\title{
GENERATORS FOR CUBIC SURFACES WITH TWO SKEW LINES OVER FINITE FIELDS
}

\author{
JENNY COOLEY
}

\begin{abstract}
Let $S$ be a smooth cubic surface defined over a field $K$. As observed by Segre [5] and Manin [3] 4], there is a secant and tangent process on $S$ that generates new $K$-rational points from old. It is natural to ask for the size of a minimal generating set for $S(K)$. In a recent paper, for fields $K$ with at least 13 elements, Siksek [7] showed that if $S$ contains a skew pair of $K$-lines then $S(K)$ can be generated from one point. In this paper we prove the corresponding version of this result for fields $K$ having at least 4 elements, and slightly milder results for $\# K=2$ or 3 .
\end{abstract}

\section{INTRODUCTION}

Let $E$ be an elliptic curve over a field $K$. It is well-known that, if $K$ is finite, then the set of $K$-rational points $E(K)$ is a finite abelian group that is either cyclic or isomorphic to a product of two cyclic subgroups. The group structure on $E$ is given by the familiar secant and tangent process.

Let $S$ be a smooth cubic surface over a field $K$. There is still a secant and tangent process that generates new points from old points. This process was introduced by Segre [5] and studied by several authors, most notably Manin [3, 4]. This process does not give the set of $K$-rational

points $S(K)$ a group structure. However, it is reasonable to ask, for $K$ a field, whether it is still possible to generate all the $K$-rational points from just one or two points. In a recent paper, Siksek [7] shows the following. Let $S$ be a smooth cubic surface defined over a field $K$ having at least 13 elements. Suppose $S$ contains a skew pair of lines $\ell_{1}, \ell_{2}$ defined over $K$. Then $S(K)$ can be generated by just one point. The purpose of this paper is to extend the proof of this to fields with at least 4 elements, and prove similar (but slightly weaker) statements over fields with 2 or 3 elements.

In the remainder of this introduction, we will give a precise definition of the secant and tangent process, and state our results. Let $K$ be field and let $S$ be a smooth cubic surface defined over $K$. It is a well-known classical theorem, due to Cayley and Salmon, that $S$ contains 27 lines defined over $\bar{K}$. Let $\ell$ be a line not contained in $S$. Then $\ell \cdot S=P+Q+R$ where $P, Q, R$ are points on $S$, counted according to multiplicity. If $P, Q \in S(K)$ and $\ell$ is a $K$-line (that is, it is defined over $K)$, then $R \in S(K)$. Of course, if $P \neq Q$, then the line $\ell$ is the secant line joining $P, Q$, and if $P=Q$ then $\ell$ is a tangent line to the surface at $P$. Let $B$ be

Date: August 1, 2021.

Key words and phrases. cubic surfaces, finite fields, secant and tangent, generating set.

The author is supported by an Engineering and Physical Sciences Research Council (EPSRC) studentship. 
a subset of $S(K)$. We define a sequence of sets

$$
B=B_{0} \subseteq B_{1} \subseteq B_{2} \subseteq \cdots \subseteq S(K)
$$

inductively as follows: a point $R \in S(K)$ belongs to $B_{n+1}$ if and only if, either $R \in B_{n}$, or there are points $P, Q \in B_{n}$ and a $K$-line $\ell$ not lying on $S$ such that $\ell \cdot S=P+Q+R$. We let $\operatorname{Span}(B)=\cup_{i=0}^{\infty} B_{i}$. In other words, $\operatorname{Span}(B) \subseteq S(K)$ is that set of points that we can obtain from $B$ via successive applications of the tangent and secant process.

An Eckardt point is a point on $S$ where three of the 27 lines meet. The main aim of this paper is to prove the following theorems.

Theorem 1. Let $K$ be a field with at least 4 elements. Let $S$ be a smooth cubic surface over $K$. Suppose $S$ contains a skew pair of lines both defined over $K$. Let $P$ be any $K$-rational point on either line that is not Eckardt. Then $\operatorname{Span}(P)=S(K)$.

Theorem 2. Let $K=\mathbb{F}_{3}$. Let $S$ be a smooth cubic surface over $K$. Suppose $S$ contains a skew pair of lines $\ell$ and $\ell^{\prime}$ defined over $K$ and that $\ell$ and $\ell^{\prime}$ each contain at most one $K$-rational Eckardt point. Then there exists a point $P \in \ell(K) \cup \ell^{\prime}(K)$ such that $\operatorname{Span}(P)=S(K)$.

Theorem 3. Let $K=\mathbb{F}_{2}$. Let $S$ be a smooth cubic surface over $K$. Suppose $S$ contains a line $\ell$ defined over $K$ that does not contain any $K$-rational Eckardt points. Then there exists a point $P \in \ell(K)$ such that $\operatorname{Span}(P)=S(K)$.

The proofs of Theorems 2 and 3 use a certain amount of exhaustive computer enumeration. It is perhaps appropriate to add a few words as to why this is not convenient for Theorem 1, especially as the results of Siksek allow us to reduce to fields having at most 11 elements. To prove Theorem 1 by exhaustive enumeration over a finite field $K$ we need to enumerate up to projective equivalence quadruples $\left(S, \ell, \ell^{\prime}, P\right)$ where $S$ is a smooth cubic surface over $K, \ell, \ell^{\prime}$ are a skew pair of $K$ lines lying on $S$, and $P \in \ell(K)$ is a non-Eckardt point. Moreover, for each of these quadruples we would want to apply the tangent and secant process repeatedly to prove that $\operatorname{Span}(P)=S(K)$. We did invest some effort into understanding the invariant theory needed for the enumeration, but it seems to us that the theory needed to make the enumeration practical for, say, $K=\mathbb{F}_{11}$, would be far more complicated than our theoretical proof of Theorem 1

\section{Preliminary Results}

Here we quote some preliminary results on the geometry and arithmetic of cubic surfaces.

Theorem 4. (Cayley-Salmon) Every non-singular cubic surface over an algebraically closed field contains exactly 27 lines.

Every line $\ell$ on the surface meets exactly 10 other lines, which break up into 5 pairs $\ell_{i}, \ell_{i}^{\prime}(i=1, \ldots, 5)$ such that $\ell, \ell_{i}$ and $\ell_{i}^{\prime}$ are coplanar, and $\left(\ell_{i} \cup \ell_{i}^{\prime}\right) \cap\left(\ell_{j} \cup \ell_{j}^{\prime}\right)=\emptyset$ for $i \neq j$.

Proof. For a proof see [2, V.4] or [6, Section IV.2].

For now $S$ will denote a smooth cubic surface in $\mathbb{P}^{3}$ over a field $K$, defined by a homogeneous cubic polynomial $F \in K\left[x_{0}, x_{1}, x_{2}, x_{3}\right]$. 
The remainder of the section summarizes some results (mostly standard) that can be found in Siksek's paper [7, Section 2]. For a point $P \in S(\bar{K})$, we shall denote the tangent plane to $S$ at $P$ by $\Pi_{P}$. This is given by $\Pi_{P}: \nabla F(P) \cdot \mathbf{x}=0$. We shall write $\Gamma_{P}$ for the plane curve $S \cdot \Pi_{P}$. It is easy to check (using the smoothness of $S$ ) that $\Gamma_{P}$ does not contain any multiple components. It is a degree 3 plane curve which is singular at $P$. If $\Gamma_{P}$ is irreducible, it is nodal or cuspidal at $P$. If $\Gamma_{P}$ is reducible then it is the union of a line and an irreducible conic, or of three distinct lines. The curve $\Gamma_{P}$ contains every $\bar{K}$-line on $S$ that passes through $P$.

A $\bar{K}$-line $\ell$ is called an asymptotic line (c.f. 8, Section 2]) at $P \in S(\bar{K})$ if $(\ell \cdot S)_{P} \geq 3$. As $S$ is a cubic surface, it is seen that for an asymptotic line $\ell$ at $P$, either $(\ell \cdot S)_{P}=3$ or $\ell \subset S$. The asymptotic lines at $P$ are contained in $\Pi_{P}$.

Any line contained in $S$ and passing through $P$ is an asymptotic line through $P$. The number of distinct asymptotic $\bar{K}$-lines at $P$ is either 1,2 or infinity. If $S$ has either 1 or infinitely many asymptotic lines at $P$ then we shall call $P$ a parabolic point. The case where there are infinitely many asymptotic lines at $P$ is special: in this case $\Gamma_{P}$ decomposes as a union of three $\bar{K}$-lines passing through $P$ lying on $S$ and so the point $P$ is an Eckardt point. If $P$ is parabolic but not Eckardt, the curve $\Gamma_{P}$ has a cusp at $P$. Note that for $P$ lying on a line $\ell \subset S$, if $P$ is not Eckardt then $\Gamma_{P}=\ell \cup C$ where $C$ is an irreducible conic, and $\ell$ lies tangent to $C$. If $P$ is non-parabolic, then $\Gamma_{P}$ has a node at $P$.

We shall also need to study the number of parabolic points on a line lying on a cubic surface. Let $\mathbb{P}^{3^{*}}$ be the dual projective space and write $\gamma: S \rightarrow \mathbb{P}^{3^{*}}$ for the Gauss map which sends a point to its tangent plane. A useful characterisation of parabolic points is that they are the points of ramification of the Gauss map [8, Section 2]. If $\ell \subset S$ and $P \in \ell$, then $\ell$ is contained in the tangent plane $\Pi_{P}$. The family of planes through $\ell$ can be identified with $\mathbb{P}^{1}$ and once such an identification is fixed we let $\gamma_{\ell}: \ell \rightarrow \mathbb{P}^{1}$ be the map that sends a point on $\ell$ to its tangent plane through $\ell$. The map $\gamma_{\ell}$ has degree 2 ([7, proof of Lemma 2.2]), and hence is separable if $\operatorname{char}(K) \neq 2$.

Lemma 1. (Siksek 7, Lemma 2.2]) Let $\ell$ be a $K$-line contained in $S$.

(i) If $\operatorname{char}(K) \neq 2$ then $\gamma_{\ell}$ is separable. Precisely two points $P \in \ell(\bar{K})$ are parabolic, and so there are at most two Eckardt points on $\ell$.

(ii) If $\operatorname{char}(K)=2$ and $\gamma_{\ell}$ is separable then there is precisely one point $P \in \ell(\bar{K})$ which is parabolic and so at most one Eckardt point on $\ell$.

(iii) If $\operatorname{char}(K)=2$ and $\gamma_{\ell}$ is inseparable then every point $P \in \ell(\bar{K})$ is parabolic and the line $\ell$ contains exactly 5 Eckardt points.

Finally we shall need the following result, which in effect says that we can restrict ourselves to fields having at most 11 elements in the proofs of Theorems 1 3

Theorem 5. (Siksek [7, Theorem 1]) Let $K$ be a field with at least 13 elements. Let $S$ be a smooth cubic surface over $K$. Suppose $S$ contains a pair of skew lines both defined over $K$. Let $P \in S(K)$ be a point on either line that is not an Eckardt point. Then $\operatorname{Span}(P)=S(K)$.

\section{Proof of Theorem 1}

Lemma 2. Let $K$ be a field with at least 4 elements and $S$ a smooth cubic surface defined over $K$. Let $\ell$ be a $K$-line on $S$. Let $P \in \ell(K)$ be a point that does not lie 
on any other line belonging to $S$. Then

$$
\ell(K) \subseteq \Gamma_{P}(K) \subseteq \operatorname{Span}(P) .
$$

Proof. The curve $\Gamma_{P}$ has degree 3 . The line $\ell$ is an irreducible component of $\Gamma_{P}$, and there are no other lines in $S$ passing through $P$. Thus $\Gamma_{P}=\ell \cup C$, where $C$ is an irreducible conic. Note $C \cdot \ell=P+P^{\prime}$ where $P^{\prime}$ is a point in $S(K)$. Note also that since $P^{\prime}$ lies on both $\ell$ and $C$, any line $\ell^{\prime} \subset \Pi_{P}, \ell^{\prime} \neq \ell$ going through $P^{\prime}$ will have a double intersection at $P^{\prime}$, i.e. $\Pi_{P}$ is the tangent plane at $P^{\prime}$ and hence $\Gamma_{P^{\prime}}=\Gamma_{P}$. We want to show that $\Gamma_{P}(K) \subseteq \operatorname{Span}(P)$.

Let $Q \in C(K), Q \neq P, P^{\prime}$. Let $\ell^{\prime}$ be the line joining $P$ and $Q$. Then $\ell^{\prime} \cdot S=$ $2 P+Q$. Thus $Q \in \operatorname{Span}(P)$. Hence $C(K) \backslash\left\{P^{\prime}\right\} \subseteq \operatorname{Span}(P)$. We now want to show that $\ell(K) \backslash\left\{P^{\prime}\right\} \subseteq \operatorname{Span}(P)$. Fix $Q \in C(K) \backslash\left\{P, P^{\prime}\right\}$, let $R \in \ell(K) \backslash\left\{P, P^{\prime}\right\}$ and let $\ell^{\prime}$ be the line joining $Q$ and $R$. Then $\ell^{\prime} \cdot S=Q+R+R^{\prime}$, where $R^{\prime} \in C(K)$. Since $Q, R^{\prime} \in C(K) \backslash\left\{P^{\prime}\right\} \subseteq \operatorname{Span}(P)$, we have $R \in \operatorname{Span}(P)$. Thus $\Gamma_{P}(K) \backslash\left\{P^{\prime}\right\} \subseteq$ $\operatorname{Span}(P)$.

If $P=P^{\prime}$, then we have $\Gamma_{P}(K) \subset \operatorname{Span}(P)$ and we are done. Suppose now that $P \neq P^{\prime}$. To complete the proof, we must show that $P^{\prime} \in \operatorname{Span}(P)$. As $P \neq P^{\prime}$ but $\Gamma_{P}=\Gamma_{P^{\prime}}$, it follows from Lemma 1 that $\gamma_{\ell}$ is separable, and that therefore the line $\ell$ contains at most two Eckardt points. Since $|K| \geq 4$, the line $\ell$ has at least five $K$-rational points, and so there is some point $R \in \ell(K)$ that is neither Eckardt, nor equal to $P, P^{\prime}$. As noted above $\Pi_{P}=\Pi_{P^{\prime}} \supset \ell \cup C$. As $\gamma_{\ell}$ has degree 2 , we see that $\Pi_{R} \neq \Pi_{P}$. There are now two cases to consider. The first is when $\Gamma_{R}=\ell \cup C^{\prime}$ where $C^{\prime}$ is an irreducible conic, and the second is when $\Gamma_{R}$ is a union of three lines. For the first case, we have

$$
\Gamma_{R}(K) \backslash\left\{R^{\prime}\right\} \subseteq \operatorname{Span}(R) \subseteq \operatorname{Span}(P)
$$

where $\ell \cdot C^{\prime}=R+R^{\prime}$. Note, $P^{\prime} \neq R^{\prime}$, as $\Pi_{P^{\prime}}=\Pi_{P} \neq \Pi_{R}=\Pi_{R^{\prime}}$. Hence $P^{\prime} \in \operatorname{Span}(P)$, and the proof is complete in this case.

Finally, we must consider the case where $\Gamma_{R}$ is the union of three lines, which must include $\ell$. Let the other two lines be $\ell_{2}$ and $\ell_{3}$, where $\ell \cdot \ell_{2}=R, \ell \cdot \ell_{3}=R^{\prime}$ and $\ell_{2} \cdot \ell_{3}=R^{\prime \prime}$. As $R$ is not Eckardt, $R, R^{\prime}$ and $R^{\prime \prime}$ are distinct. Since $\ell$ and $R$ are $K$-rational, so are the lines $\ell_{2}, \ell_{3}$ and the points $R^{\prime}$ and $R^{\prime \prime}$.

First note that both $R$ and $R^{\prime}$ are in $\operatorname{Span}(P)$ as they both lie on $\ell$ and are not equal to $P^{\prime}$. Let $Q \in \ell_{3}(K), Q \neq R^{\prime}, R^{\prime \prime}$. Let $m$ be the line joining $R$ and $Q$. Then $m \cdot S=2 R+Q$, and so $Q \in \operatorname{Span}(P)$. Thus

$$
\ell_{3}(K) \backslash\left\{R^{\prime \prime}\right\} \subseteq \operatorname{Span}(P)
$$

and likewise

$$
\ell_{2}(K) \backslash\left\{R^{\prime \prime}\right\} \subseteq \operatorname{Span}(P) .
$$

Take $Q \in \ell_{3}(K), Q \neq R^{\prime}, R^{\prime \prime}$. Let $m$ be the line joining $P^{\prime}$ and $Q$. Then $m \cdot S=$ $P^{\prime}+Q+Q^{\prime}$, where $Q^{\prime} \in \ell_{2}(K), Q^{\prime} \neq R, R^{\prime \prime}$. Thus $P^{\prime} \in \operatorname{Span}(P)$, completing the proof.

The following lemma is a strengthening of Lemma 2 ,

Lemma 3. Let $K$ be a field with at least 4 elements and $S$ a smooth cubic surface defined over $K$. Let $\ell$ be a $K$-line on $S$. Let $P \in \ell(K)$ and suppose that $P$ is not an Eckardt point. Then

$$
\ell(K) \subseteq \Gamma_{P}(K) \subseteq \operatorname{Span}(P) .
$$


Proof. Let $P \in \ell(K)$ be a non-Eckardt point. If $P$ does not lie on any other line contained in $S$ then we can invoke Lemma 2. Thus we may suppose that $P$ lies on some other line $\ell_{2}$. This is necessarily a $K$-line because if it were not, its conjugate line would also pass through $P$, meaning that $P$ were an Eckardt point, which would contradict the hypotheses of the lemma. Now $\Gamma_{P}=\ell \cup \ell_{2} \cup \ell_{3}$, where $\ell_{3}$ is also a $K$-line. As $P$ is not Eckardt, $\ell_{3}$ does not pass through $P$. Let $\ell \cdot \ell_{3}=P^{\prime}$ and $\ell_{2} \cdot \ell_{3}=P^{\prime \prime}$. As before

$$
\ell_{3}(K) \backslash\left\{P^{\prime}, P^{\prime \prime}\right\} \subseteq \operatorname{Span}(P) .
$$

As in the proof of Lemma 2 , $\gamma_{\ell_{3}}$ is separable, and so by Lemma 1, there are at most two Eckardt points on $\ell_{3}$. Since $\ell_{3}(K)$ has at least 5 points, we see that there is some $Q \in \ell_{3}(K) \backslash\left\{P^{\prime}, P^{\prime \prime}\right\}$ that is not Eckardt. We consider two cases. The first is where $Q$ does not lie on any other line. Then, by Lemma 2, $\ell_{3}(K) \subseteq \operatorname{Span}(Q) \subseteq \operatorname{Span}(P)$. Thus $P, P^{\prime}, P^{\prime \prime} \in \operatorname{Span}(P)$. As before, we can generate the remaining points in $\Gamma_{P}(K)=\ell(K) \cup \ell_{2}(K) \cup \ell_{3}(K)$, from these.

The remaining case is when $Q$ lies on some other line $\ell_{4}$ and so $\Gamma_{Q}=\ell_{3} \cup \ell_{4} \cup \ell_{5}$. Just as in the argument at the end of the proof of Lemma 2, we can show that $P$, $P^{\prime}$ and $P^{\prime \prime}$ are in $\operatorname{Span}(P)$ and complete the proof.

The following propositions will be useful.

Proposition 1. Let $K$ be a field with at least 4 elements and $S$ be a smooth cubic surface defined over $K$. Suppose $S$ contains a skew pair of lines $\ell, \ell^{\prime}$ and there is a non-Eckardt point $P \in \ell(K)$ such that $\Pi_{P} \cdot \ell^{\prime}$ is also non-Eckardt. Then

$$
\ell^{\prime}(K) \subseteq \operatorname{Span}(\ell(K)) .
$$

Proof. Let $Q=\Pi_{P} \cdot \ell^{\prime}$. Note that $Q \in \Gamma_{P}(K)$, thus $Q \in \operatorname{Span}(P) \subseteq \operatorname{Span}(\ell(K))$ by Lemma 3 . Suppose $Q$ is non-Eckardt. Applying Lemma 3 again we have

$$
\ell^{\prime}(K) \subseteq \operatorname{Span}(Q) \subseteq \operatorname{Span}(\ell(K)) .
$$

Proposition 2. Let $S$ be a smooth cubic surface defined over a field $K$. Suppose $S$ contains a skew pair of $K$-lines $\ell, \ell^{\prime}$ and let $P \in \ell(K)$. Let $\Gamma_{P}$ be the union of $\ell$ and an irreducible conic. Then the point $\ell^{\prime} \cdot \Pi_{P}$ is not an Eckardt point.

Proof. Let $P \in \ell(K)$. Let $E=\ell^{\prime} \cdot \Pi_{P}$. Suppose $\Gamma_{P}=C \cup \ell$ where $C$ is an irreducible conic. We will first show that as $C$ is irreducible, it must be absolutely irreducible. Indeed, suppose $C=m \cup n$ where $m, n$ are $\bar{K}$-lines, that are Galois conjugates. The $K$-point $P$ lies on one of them and hence both. Now $E$ is a $K$-point, and $E$ belongs to $\Gamma_{P}=\ell \cup m \cup n$ as well as $\ell^{\prime}$. Since $\ell$ and $\ell^{\prime}$ are skew, without loss of generality $E \in m$. Hence the line $m$ joins the $K$-points $P$ and $E$ and is therefore defined over $K$. This contradicts the irreducibility of $C$. Hence $C$ is an absolutely irreducible conic.

We must prove that $E$ is not an Eckardt point. Suppose it is. Let $\ell_{2}$ and $\ell_{3}$ be the other two lines going through $E$. Let $Q=\ell \cdot \Pi_{E}$, then without loss of generality $Q=\ell \cdot \ell_{2}$. Note that $\ell_{2}$ must be in the tangent plane to $S$ at $Q$, so $\Pi_{Q} \neq \Pi_{P}$ since $\Gamma_{P}=\ell \cup C$. Note also that $E=\ell^{\prime} \cdot \Pi_{Q}$, which implies that $\Pi_{Q}$ is the unique plane containing $\ell$ and $E$. However, the plane $\Pi_{P}$ also contains $\ell$ and $E$. But $\Pi_{P} \neq \Pi_{Q}$ so we have reached a contradiction, and the point $\ell^{\prime} \cdot \Pi_{P}$ cannot be an Eckardt point. 
Lemma 4. Let $K$ be a field with at least 7 elements and $\operatorname{char}(K) \neq 2$. Let $S$ be a smooth cubic surface defined over $K$. Suppose $S$ contains a skew pair of $K$-lines $\ell$, $\ell^{\prime}$. Then

$$
\ell^{\prime}(K) \subseteq \operatorname{Span}(\ell(K)) .
$$

Proof. By Lemma 1, there are at most two $K$-rational Eckardt points on each of $\ell$, $\ell^{\prime}$. Hence

$$
\#(\ell(K) \backslash\{\text { Eckardt points }\}) \geq 6 .
$$

The Gauss map on $\ell$ has degree 2 so

$$
\# \gamma_{\ell}(\ell(K) \backslash\{\text { Eckardt points }\}) \geq 3 .
$$

Therefore we must have a non-Eckardt $P \in \ell(K)$ mapping to a plane $\gamma_{\ell}(P)$ that intersects $\ell^{\prime}$ in a non-Eckardt point $Q$. We invoke Proposition 1 to obtain $\ell^{\prime}(K) \subseteq$ $\operatorname{Span}(\ell(K))$, which completes the proof.

Lemma 5. Let $K$ be $\mathbb{F}_{4}, \mathbb{F}_{5}$ or $\mathbb{F}_{8}$ and $S$ be a smooth cubic surface defined over $K$. Suppose $S$ contains a skew pair of $K$-lines $\ell, \ell^{\prime}$. Let $P \in \ell(K)$ be a point that is not Eckardt. Then

$$
\ell_{2}(K) \subseteq \operatorname{Span}\left(\ell_{1}(K)\right)
$$

where $\ell_{1}, \ell_{2}$ is a skew pair of $K$-lines in $S$ that may or may not be equal to $\ell, \ell^{\prime}$.

Proof. First note that for any non-Eckardt point $P \in \ell(K)$ we have $\ell(K) \subseteq \operatorname{Span}(P)$ by Lemma 3. Suppose $P \in \ell(K)$ is a point such that $\Gamma_{P}=C \cup \ell$ where $C$ is an irreducible conic. Then $Q=\Pi_{P} \cdot \ell^{\prime}$ is a non-Eckardt point by Proposition 2 We invoke Lemma 3 to obtain

$$
\ell^{\prime}(K) \subseteq \operatorname{Span}(Q) \subseteq \operatorname{Span}(P) \subseteq \operatorname{Span}(\ell(K)) .
$$

Therefore we assume that all points in $\ell(K)$ lie on at least one $K$-line in $S$ other than $\ell$. Note that this excludes the case where $\operatorname{char}(K)=2$ and $\gamma_{\ell}$ is inseparable, since in such cases we must have at least one non-Eckardt point in $\ell(K)$ and by Lemma 1 any such $P$ must be parabolic, and hence $\Gamma_{P}$ is the union of $\ell$ and an irreducible conic. Thus we may assume that $\gamma_{\ell}$ is separable. In which case we must have four points $P, P^{\prime}, R, R^{\prime} \in \ell(K)$ with $\Gamma_{P}=\Gamma_{P^{\prime}}, \Gamma_{R}=\Gamma_{R^{\prime}}$. Let $\ell_{1} \subseteq S$ be the $K$-line such that $\ell \cdot \ell_{1}=P$ and $\ell_{2} \subseteq S$ be the $K$-line such that $\ell \cdot \ell_{2}=R$. Then $l_{1} \subseteq \Pi_{P}$ and $\ell_{2} \subseteq \Pi_{R}$ and so $\ell_{1}$ is skew to $\ell_{2}$. Note that $P$ is a non-Eckardt point on $\ell_{1}$ so $\ell_{1}(K) \subseteq \operatorname{Span}(P)$ and likewise $\ell_{2}(K) \subseteq \operatorname{Span}(R) \subseteq \operatorname{Span}(P)$. Hence

$$
\ell_{1}(K) \cup \ell_{2}(K) \subseteq \operatorname{Span}(P),
$$

which completes the proof.

The following lemma is stated in 7 , with the hypothesis that $K$ has at least 13 elements. Using our Lemma 3 and by modifying the proof we can strengthen this as follows.

Lemma 6. Let $K$ be a field with at least 4 elements, and let $S$ be a smooth cubic surface defined over $K$. Suppose $S$ contains a pair of skew lines $\ell_{1}$ and $\ell_{2}$ both defined over $K$. If $\# K=4$, then suppose also that at least one of them contains a non-Eckardt K-point. Then

$$
\operatorname{Span}\left(\ell_{1}(K) \cup \ell_{2}(K)\right)=S(K) .
$$


Proof. Let $P$ be a $K$-point on $S$ not belonging to either line; we will show that $P$ belongs to the span of $\ell_{1}(K) \cup \ell_{2}(K)$. Let $\Pi_{1}$ be the unique plane containing $\ell_{2}$ and $P$, and $\Pi_{2}$ the unique plane containing $\ell_{1}$ and $P$. Since $\ell_{1}$ and $\ell_{2}$ are skew we know that $\ell_{i} \not \subset \Pi_{i}$. Write $Q_{i}=\ell_{i} \cap \Pi_{i}$. Note that $P, Q_{1}$ and $Q_{2}$ are distinct points on $S$ that also belong to the $K$-line $\ell=\Pi_{1} \cap \Pi_{2}$. Suppose first that $\ell \not \subset S$. Then $\ell \cdot S=P+Q_{1}+Q_{2}$. Thus $P \in \operatorname{Span}\left(\ell_{1}(K) \cup \ell_{2}(K)\right)$ as required.

Next suppose that $\ell \subset S$. If $|K| \geq 5$, we know from Lemma 1 that there are non-Eckardt $K$-points on both $\ell_{1}$ and $\ell_{2}$. If $|K|=4$, then one of the hypotheses of the lemma is that there is a non-Eckardt $K$-point on one of those two lines. Without loss of generality, $R \in \ell_{2}(K)$ is non-Eckardt.

Now $\ell \subset \Gamma_{Q_{1}}$. If $Q_{1}$ is not Eckardt, then by Lemma 3 ,

$$
P \in \ell(K) \subseteq \Gamma_{Q_{1}}(K) \subseteq \operatorname{Span}\left(Q_{1}\right) \subseteq \operatorname{Span}\left(\ell_{1}(K)\right) .
$$

Thus we may assume that $Q_{1}$ is Eckardt. Similarly $Q_{2}$ is Eckardt. Then $\Gamma_{Q_{1}}=$ $\ell \cup \ell_{1} \cup \ell_{3}$ where $\ell_{3}$ is also $K$-rational. Now $\ell_{2}$ must meet that tangent plane $\Pi_{Q_{1}}$ in a unique point, and that is $Q_{2} \in \ell$. Therefore, $\ell_{2}$ and $\ell_{3}$ are skew. Consider $\gamma_{\ell_{2}}$. As $Q_{2}$ is Eckardt, it is a ramification point for $\gamma_{\ell_{2}}$. Therefore $\gamma_{\ell_{2}}\left(Q_{2}\right) \neq \gamma_{\ell_{2}}(R)$. Note $\gamma_{\ell_{2}}\left(Q_{2}\right) \cdot \ell_{3}=Q_{1}$, so $\gamma_{\ell_{2}}(R) \cdot \ell_{3}=R^{\prime}$ where $R^{\prime}$ is a $K$-point distinct from $Q_{1}$. Moreover, $R^{\prime} \in \operatorname{Span}(R) \subseteq \operatorname{Span}\left(\ell_{2}(K)\right)$. Finally, consider the line that joints $R^{\prime}$ and $P$. This lies in $\Pi_{Q_{1}}$, but not on $S$, and so must intersect $\ell_{1}$ in a $K$-point $R^{\prime \prime}$. Hence $P \in \operatorname{Span}\left(\ell_{1}(K) \cup \ell_{2}(K)\right)$ which completes the proof.

Proof of Theorem 1, If $K$ has 13 or more elements then we can invoke Siksek's Theorem 5. Thus we may restrict our attention to the cases $\# K=4,5,7,8,9$, 11. The proof follows from Lemmas 3, 4, 5 and 6.

\section{Proof of Theorem 2}

Lemma 7. Let $K=\mathbb{F}_{3}$ and $S$ be a smooth cubic surface defined over $K$. Suppose $S$ contains a skew pair of $K$-lines $\ell, \ell^{\prime}$ and suppose $\ell$ contains exactly one $K$-rational Eckardt point. Then there exists a non-Eckardt point $P \in \ell(K)$ such that

$$
S(K)=\operatorname{Span}(P) .
$$

Proof. The lemma was proved by an exhaustive computer enumeration implemented in MAGMA [1. By a projective change of co-ordinates we may first suppose that the line $\ell$ is defined by $X=Y=0$, and that therefore the surface $S$ has the form $X Q_{1}+Y Q_{2}$ where $Q_{1} \in \mathbb{F}_{3}[X, Y, Z, W]$ and $Q_{2} \in \mathbb{F}_{3}[Y, Z, W]$ are homogeneous quadratic forms. We may then by further projective changes of co-ordinates suppose that the $K$-rational Eckardt point on $\ell$ is the point $P=(0: 0: 0: 1)$. We denote the other two lines in $S$ passing through $P$ by $\ell_{1}, \ell_{2}$. The line $\ell^{\prime}$ must intersect the plane $\Pi_{P}$ in some $K$-point $Q \neq P$. As $\ell$ and $\ell^{\prime}$ are skew, $Q \notin \ell$ and so without loss of generality $Q \in \ell_{1}$. The line $\ell_{1}$ joins two $K$-points and is therefore a $K$-line. Hence $\ell_{2}$ is also a $K$-line. By yet another change of coordinates that preserves $\ell$ and $P$, we may suppose that $\ell_{1}$ and $\ell_{2}$ have the equations $\ell_{1}: X=Z=0$ and $\ell_{2}: X=Y+Z=0$. Thus every cubic surface defined over $\mathbb{F}_{3}$ containing a skew pair of $K$-lines has a model that can be written in the form

$$
X\left(a X^{2}+b X Y+c X Z+d Y^{2}+e Y+f Z^{2}+g W^{2}\right)+Y Z(Y+Z)
$$

where $a, \ldots, g \in \mathbb{F}_{3}$. Therefore the program was enumerated over $3^{7}=2187$ cubic surfaces. Our program checked the surfaces for smoothness, then whether there 
was a point $P \in \ell$ such that $\operatorname{Span}(P)=S(K)$. In the cases where this failed, we verified that there was a second Eckardt point in $\ell(K)$.

The remaining cases in which there are no $K$-rational Eckardt points on either $\ell, \ell^{\prime}$ result from the following lemmas.

Lemma 8. Let $K=\mathbb{F}_{3}$ and $S$ be a smooth cubic surface defined over $K$. Let $\ell$ be a $K$-line on $S$ that does not contain any $K$-rational Eckardt points. Let $P \in \ell(K)$ be a point that does not lie on any other line belonging to $S$. Then

$$
\ell(K) \subseteq \Gamma_{P}(K) \subseteq \operatorname{Span}(P) .
$$

Proof. If $P$ is a parabolic point then, as in the proof of Lemma 2, we know that $\Gamma_{P}(K) \subseteq \operatorname{Span}(P)$. Otherwise there is a point $P^{\prime} \in \ell(K)$ such that $P^{\prime} \neq P$ but $\Gamma_{P^{\prime}}=\Gamma_{P}$. In this case, similarly to the proof of Lemma 2, we have

$$
\Gamma_{P}(K) \backslash\left\{P^{\prime}\right\} \subseteq \operatorname{Span}(P) .
$$

As $K=\mathbb{F}_{3}$ there are four points in $\ell(K), P, P^{\prime}, R$ and $R^{\prime}$. If $\Gamma_{R}=\ell \cup C$ where $C$ is an irreducible conic then $P^{\prime} \in \operatorname{Span}(R) \subseteq \operatorname{Span}(P)$. Hence $\Gamma_{P}(K) \subseteq \operatorname{Span}(P)$. Otherwise $\Gamma_{R}=\Gamma_{R^{\prime}}$ and is the union of $3 K$-lines in $S$, which are $\ell, \ell_{2}$ and $\ell_{3}$, where $\ell \cdot \ell_{2}=R, \ell \cdot \ell_{3}=R^{\prime}$ and $\ell_{2} \cdot \ell_{3}=R^{\prime \prime}$. We know that $\left(\ell_{2}(K) \cup \ell_{3}(K)\right) \backslash\left\{R^{\prime \prime}\right\} \subseteq$ $\operatorname{Span}\left(R, R^{\prime}\right)$ and $P^{\prime} \in \operatorname{Span}\left(\left(\ell_{2}(K) \cup \ell_{3}(K) \backslash\left\{R^{\prime \prime}\right\}\right)\right.$. Thus

$$
\ell(K) \subseteq \Gamma_{P}(K) \subseteq \operatorname{Span}(P)
$$

which completes the proof.

Lemma 9. Let $K=\mathbb{F}_{3}$ and $S$ be a smooth cubic surface defined over $K$. Suppose $S$ contains a skew pair of $K$-lines, $\ell, \ell^{\prime}$ that contains no $K$-rational Eckardt points. Then there is a point $P \in \ell(K)$ such that

$$
\ell(K) \subseteq \Gamma_{P} \subseteq \operatorname{Span}(P) .
$$

Proof. If there is a point in $\ell(K)$ that lies on no other line in $S$ then the result follows from Lemma 8 . So suppose every point in $\ell(K)$ lies on exactly one other $K$-line in $S$. Since $K=\mathbb{F}_{3}$ there are 4 points in $\ell(K)$, which we denote $P, P^{\prime}, R$, $R^{\prime}$. We have $\Gamma_{P}=\Gamma_{P^{\prime}}=\ell \cup \ell_{1} \cup \ell_{2}$ with $P=\ell \cdot \ell_{1}, P^{\prime}=\ell \cdot \ell_{2}$ and $\Gamma_{R}=\Gamma_{R^{\prime}}$ with $R=\ell \cdot \ell_{3}, R^{\prime}=\ell \cdot \ell_{4}$. Let $P^{\prime \prime}=\ell_{1} \cdot \ell_{2}$. By the argument in the proof of Lemma 7 we know that $\ell^{\prime}$ intersects precisely one of $\ell_{1}, \ell_{2}$ and one of $\ell_{3}, \ell_{4}$. Without loss of generality suppose that $\ell^{\prime}$ intersects $\ell_{2}$ and $\ell_{4}$. By our hypotheses $\ell^{\prime}$ contains no $K$-rational Eckardt points, therefore the point $Q=\ell^{\prime} \cdot \ell_{2}$ is nonEckardt. Note that $Q \in\left(\ell_{2}(K) \backslash\left\{P^{\prime}, P^{\prime \prime}\right\}\right) \subseteq \operatorname{Span}(P)$. Let $Q^{\prime}$ be the remaining point in $\ell_{2}(K) \backslash\left\{P^{\prime}, P^{\prime \prime}\right\}$. Our aim is the show that $P^{\prime}, P^{\prime \prime} \in \operatorname{Span}(P)$ since we can generate all the remaining points in $\ell(K), \ell_{1}(K)$ from $P^{\prime \prime}, P^{\prime}$ respectively. If $Q$ is a parabolic point then

$$
P^{\prime}, P^{\prime \prime} \in \ell_{2}(K) \subseteq \operatorname{Span}(Q) \subseteq \operatorname{Span}(P) .
$$

Likewise if $\Gamma_{Q}=\Gamma_{Q^{\prime}}$ then

$$
P^{\prime}, P^{\prime \prime} \in \ell_{2}(K) \subseteq \operatorname{Span}\left(Q, Q^{\prime}\right) \subseteq \operatorname{Span}(P),
$$

which completes the proof. 
Lemma 10. Let $K=\mathbb{F}_{3}$ and $S$ be a smooth cubic surface defined over $K$. Suppose $S$ contains a skew pair of $K$-lines $\ell, \ell^{\prime}$ that contains no $K$-rational Eckardt points. Then

$$
\ell^{\prime}(K) \subseteq \operatorname{Span}(\ell(K))
$$

Proof. Let $P \in \ell(K)$. By Lemma $9 Q=\Pi_{P} \cdot \ell^{\prime} \in \Gamma_{P} \subseteq \operatorname{Span}(P)$. We invoke Lemma 9 again to obtain

$$
\ell^{\prime}(K) \subseteq \operatorname{Span}(Q) \subseteq \operatorname{Span}(P) \subseteq \operatorname{Span}(\ell(K)) .
$$

Lemma 11. Let $K=\mathbb{F}_{3}$, and let $S$ be a smooth cubic surface defined over $K$. Suppose $S$ contains a skew pair of $K$-lines $\ell_{1}, \ell_{2}$, which contains no $K$-rational Eckardt points. Then

$$
\operatorname{Span}\left(\ell_{1}(K) \cup \ell_{2}(K)\right)=S(K) .
$$

Proof. This proof is similar to the proof of Lemma 6 . Let $P$ be a $K$-point on $S$ not belonging to either line; we will show that $P$ belongs to the span of $\ell_{1}(K) \cup \ell_{2}(K)$. Let $\Pi_{1}$ be the unique plane containing $\ell_{2}$ and $P$, and $\Pi_{2}$ the unique plane containing $\ell_{1}$ and $P$. Since $\ell_{1}$ and $\ell_{2}$ are skew we know that $\ell_{i} \not \subset \Pi_{i}$. Write $Q_{i}=\ell_{i} \cap \Pi_{i}$. Note that $P, Q_{1}$ and $Q_{2}$ are distinct points on $S$ that also belong to the $K$-line $\ell=\Pi_{1} \cap \Pi_{2}$. Suppose first that $\ell \not \subset S$. Then $\ell \cdot S=P+Q_{1}+Q_{2}$. Thus $P \in \operatorname{Span}\left(\ell_{1}(K) \cup \ell_{2}(K)\right)$ as required.

Next suppose that $\ell \subset S$. Now $\ell \subset \Gamma_{Q_{1}}$. Since $Q_{1}$ is not Eckardt, then by Lemma 9

$$
P \in \ell(K) \subseteq \Gamma_{Q_{1}}(K) \subseteq \operatorname{Span}\left(Q_{1}\right) \subseteq \operatorname{Span}\left(\ell_{1}(K)\right),
$$

which completes the proof.

Proof of Theorem Q. The proof follows from Lemmas 9, 10, 11 and 7 ,

\section{Proof of Theorem 3}

Theorems 3 was proved by an exhaustive computer enumeration implemented in MAGMA [1].

Proof of Theorem 3. By a projective change of coordinates we may suppose that the line $\ell$ is defined by $X=Y=0$, and that therefore the surface $S$ has the form $X Q_{1}+Y Q_{2}$ where $Q_{1} \in \mathbb{F}_{2}[X, Y, Z, W]$ and $Q_{2} \in \mathbb{F}_{2}[Y, Z, W]$ are homogeneous quadratic forms. Our program enumerated all possible $Q_{1}, Q_{2}$, checked the surface for smoothness and whenever $\ell$ contained no $K$-rational Eckardt points, it verified that the span of one of its $K$-points is equal to $S(K)$. This meant the program was enumerated over $2^{16}=65536$ possible models, as there are 10 monomials in $X, Y$, $Z, W$, and 6 monomials in $Y, Z, W$.

\section{REFERENCES}

[1] W. Bosma, J. Cannon and C. Playoust, The Magma Algebra System I: The User Language, J. Symb. Comp. 24 (1997), 235-265. (See also http://magma.maths.usyd.edu.au/magma/)

[2] R. Hartshorne, Algebraic Geometry, Springer Verlag, GTM 52, 1977.

[3] Yu. I. Manin, Cubic Forms: Algebra, Geometry, Arithmetic, North-Holland, 1974 and 1986.

[4] Yu. I. Manin, Mordell-Weil problem for cubic surfaces, pages 313-318 of Advances in mathematical sciences: CRM's 25 years (Montreal, PQ, 1994), CRM Proc. Lecture Notes 11, Amer. Math. Soc., Providence, RI, 1997. 
[5] B. Segre, A note on arithmetical properties of cubic surfaces, J. London Math. Soc. 18 (1943), $24-31$.

[6] I. R. Shafarevich, Basic Algebraic Geometry 1, second edition, Springer-Verlag, 1994.

[7] S. Siksek, On the number of Mordell-Weil generators for cubic surfaces, Journal of Number Theory 132 (2012), 2610-2629.

[8] J. F. Voloch, Surfaces in $\mathbb{P}^{3}$ over finite fields, pages 219-226 of Topics in algebraic and noncommutative geometry (Luminy/Annapolis, MD, 2001), Contemp. Math. 324, Amer. Math. Soc., Providence, RI, 2003.

Mathematics Institute, University of Warwick, Coventry, CV4 7AL, UK

E-mail address: j.a.cooley@warwick.ac.uk 